\title{
Racial Differences in Encounter Rates for Otitis Media
}

\author{
PATRICIA J. BUSH ${ }^{1+1+1}$ AND DAVID L. RABIN \\ Department of Community and lamily Medicine. Georgeton'n University School of Medicine, \\ Washington. I). (.. USA
}

\begin{abstract}
Summary
In 1975-1976, an experiment was performed at three sites of an HMO to investigate the effect of a protocol on drug prescribing for otitis media. A large and unexpected difference in encounter rates for otitis media was found among the sites. In the first 6 months of 1975, the rate of children less than 15 years with at least one encounter for otitis media at site $E_{1}$ was 155 per 1000 children. compared to 15 per 1000 at site $E_{11}$. During the study period, most of the patients at site $E_{11}$ were black. The purpose of this paper is to alert readers to factors that affect the rates of disease in clinical practice, to encourage others to suggest reasons for the difference, and to encourage research. Several explanations were investigated to explain the differences: (1) miscoding; (2) age differences; (3) payment status: (4) diagnostic style; (5) differences in encounters per episode; and (6) social differences. Encounters of purulent otitis media were probably not systematically miscoded. The age distribution within the sites was almost identical. The difference in the encounter rates was even greater for children le:'s than 15 years when only the enrolled population was examined. National incidence rates of otitis media showed rates for all ages for whites were $4.1 \%$ and for blacks $2.5 \%$. The average number of encounters per episode was 1.4 at all of the study sites. Enrolled children at site $E_{1}$ had higher encounter rates for all reasons than children at site $E_{11}$. Differences in diagnostic style may account for part of the difference in otitis media between the sites. Differences in encounter rates may also be due to real differences in disease occurrence between blacks and whites, differences in the perception of ear infection, and differences in the tendency to visit a physician for ear problems.
\end{abstract}

\section{Speculation}

Large differences in encounter rates for otitis media between blacks and whites were partially explained and raise related questions such as: (1) what is the real difference in the rates of otitis media between blacks and whites? (2) how much variation in the encounter rates for this common disorder, which leads to prescribing expensive and potentially hazardous drugs, is due to variations in physician diagnosis? (3) what can be done to rationalize diagnostic styles? (4) did the patients at site $E_{I}$ receive too much care, or did the patients at site $E_{11}$ receive too little care?

In 1975-1976, an experiment was performed at three centers of a health maintenance organization (HMO), the (ieorgetown University Community Health Plan. currently serving over 50,000 persons in the Washington. D. C'. metropolitan area. to investigate the effect of a protocol on drug prescribing for otitis media (2). During the process of the research, a large and unexpected difference in encounter rates for otitis media was found among the sites. The difference in the rates was studied to the extent possible to be certain it did not derive from an error in the study methods. Some. but not all of the difference could be explained. The purpose of this paper is to alert readers to factors that affect the rates of disease in clinical practice, to encourage others to suggest reasons for the difference, and to encourage further research.

\section{MATERIALS AND METHOIS}

To facilitate selection of a problem for which a drug therapy protocol was to be developed, the encounter rates for all of the problems presented to two of the three sites of the HMO in 1974 were obtained through a computerized system. One of the experimental sites $\left(E_{1}\right)$ is in Reston. Virginia. an affluent white-collar new town within commuting distance of the District of Columbia. and the other $\left(E_{I I}\right)$ is in a blue-collar area of Northeast $W$ ashington. The control site $(C)$ is in the middle-class Washington suburb of Kensington. MD. During the study period. nearly all of the patients at site $E_{11}$ were black. All encounters were coded by the health care providers at the HMO sites using a modification of the Royal College of Cieneral Physicians' Classification of morbidity.

Based on the frequency of its presentation and their concern with appropriate therapy. physicians at site $E_{1}$ selected otitis media for which to develop the drug therapy protocol. The protocol was developed at site $E_{1}$ and adopted for use at both sites $E_{1}$ and $E_{11}$. To evaluate the effect of the protocol. the drug prescribing for otitis media at sites $E_{1}$ and $E_{11}$ were compared with each other and with the control site $(C)$. The research design called for a comparison of drug prescribing for otitis media for the 6 months period before the protocol was developed with a 6 months postprotocol period. However. because of the unexpected paucity of otitis media encounters at $E_{I I}$, an investigation was undertaken to explain the differences in encounter rates among the sites.

\section{RESULTS}

The data obtained for the 1974 encounters at sites $E_{1}$ and $E_{11}$ indicated that purulent otitis media ranked fourth at site $E_{1}$ forming $3.9 \%$ of all encounters and 11 th at site $F_{11}$. forming $1.5 \%$ of all encounters. In the first 6 months of 1975 (the study's preprotocol phase). the rate of children less than 15 years with at least one encounter for purulent otitis media at site $E_{1}$ was 155 per 1000 children (enrollees plus fee for service) compared to 15 per 1000 at site $E_{11}$. In terms of all 1975 encounters. 150 per 1000 encounters were for purulent otitis media at site $E_{1}$ compared to 25 per 1000 at site Ell for children less than 15 years.

A number of possible explanations were investigated to explain the difference in the 1974 encounter rates for otitis media at sites $E_{1}$ and $E_{11}$ and the low number of encounters found at $E_{11}$ relative to $E_{1}$. during the study phases. These included the following: (1) miscoding: (2) age differences: (3) payment status differences: (4) diagnostic style differences: (5) differences in encounters per episode: and (6) social differences. 
MISCOI)ING;

To investigate the possibility that the differences in rates of purulent otitis media between sites $E_{1}$ and $E_{11}$ resulted from miscoding purulent as serous or chronic otitis, 1976 data were compared for children under 15 years as shown in Table 1 .

The data in Table 1 indicate that encounters of purulent otitis media were probably not systematically miscoded as serous or chronic otitis. When asked, the physicians at site $E_{11}$ claimed they used the correct codes for the three categories. Although the 1976 encounter rates for purulent otitis media at sites $E_{1}$ and $E_{11}$ were lower than during the tirst 6 months of 1975. they were significantly different from each other. The rates at site $C$ were between those at sites $E_{1}$ and $E_{11}$

\section{A(it) DIFFIRI:NCIS}

The fifteen-fold difference in encounter rates between sites $E_{t}$ and $E_{11}$ might logically have been accounted for by variations in ages within the category 0 to 14 years. If considerably more children under 5 years old were patients at site $E_{1}$ than at site $E_{11}$, higher rates of otitis would have been expected at site $E_{1}$. However. there were no significant differences in the age distributions: $10.00^{\prime \prime} ;$ of enrollees were under 5 years at site $E_{1}$ compared to $9.9 \%$ at site $E_{11}$. $1.6 \%$ were less than one year at site $E_{1}$ compared to $1.5 \%$ at site $E_{1 I}$.

\section{PAYMINT SIATIS}

Because of their lower economic status, the fee-for-service patients might be less likely to visit site $E_{11}$ than site $E_{1}$. However. the difference in the encounter rates between the two sites was even greater for children under 15 years when only the enrolled population was considered. Eighteen percent of the enrolled children less than 15 years at site $E_{1}$ had at least one encounter for purulent otitis media in the first six months of 1975, compared to only I"; at site $E_{11}$.

\section{DIAC INOSTIC STYIL DIFHERENCES}

The providers at sites $E_{1}$ and $E_{I]}$ were sent questionnaires at the end of the study period inquiring into diagnostic criteria relating to both signs and symptoms for purulent otitis. A $10 \times 10$ table format was used. and the provider was asked to check the square indicated if ( $s$ )he tended to diagnose purulent otitis in a combination of any two of the following criteria: (1) recent upper respiratory infection: (2) ear pain: (3) fever $\geq 100^{\circ} \mathrm{F}$ : (4) injected tympanic membrane: (5) absent light reflex: (6) bulging tympanic membrane: (7) positive cervical lymphadenopathy: (8) grey tympanic membrane: (9) decreased movement on pneumatoscopy: and (10) visible fluid level hehind eardrum. Six providers at site $E_{1}$ and three at site $E_{11}$. of fifteen who diagnosed otitis media in four or more patients during the study's postphase. responded. There were $115\left(43.7{ }^{\prime \prime}\right)$ of 270 possible "yeses" at site $E_{1}$ and 39 (28.9") "yeses" of a possible 135 at site $E_{11}(z=2.88: P=0.04)$. The range of "yeses" varied from 4 at site $E_{I 1}$ to 27 at site $E_{I}$ of the possible 45. Thus, differences in diagnostic style may account for part of the difference in rates of purulent otitis media between the sites.

\section{DIFFERENCES IN ENCOUNTIRS PER FPISODE}

The differences in encounter rates might have been due to differences in the average number of encounters per episode (13) if physicians at site $E_{1}$ were more likely than physicians at site $E_{I I}$ to ask children with otitis media to return for followup visits or if the children attending site $E_{1}$ were more compliant with such requests. Shurin et al. (12) found that white children visited more often after otitis media was diagnosed than biack children. However, the average number of encounters per episode of otitis was a surprisingly stable 1.4 during the postphase of all three of the study sites. indicating that variations in encounter rates represented variations in diagnosed episodes among the centers.
Table I. Total encounters and percentage of encoumters for otitis media for children under 15 vears by HMO site (1976)

\begin{tabular}{|c|c|c|c|c|}
\hline \multirow{2}{*}{ Site } & \multirow{2}{*}{ Total encounters } & \multicolumn{3}{|c|}{ Otitis mediat $(" i)$} \\
\hline & & Purulent & Serous & ('hronic \\
\hline $\mathbf{E}_{i}$ & 17.220 & 10.7 & 4.2 & 0.2 \\
\hline$E_{11}$ & 4.548 & 2.0 & 0.1 & 0.1 \\
\hline$c^{\prime}$ & 6,883 & 6.1 & 1.1 & 0.7 \\
\hline
\end{tabular}

SOCIAL DIIFERINCES

The difference in encounter rates per episode of otitis media might simply have been due to a difference in overall visit rates and thus not specifically related to otitis media. Enrolled children at site $E_{1}$ did have significantly higher encounter rates for all reasons than children at site $E_{11}$. For enrolled children under 15 years in the first 3 months of 1975 , the encounter rates at site $E_{I}$ were 1012 per 1000 compared to 377 per 1000 children at site $E_{11}$. a more than two-and-one-half-fold difference.

A 1974 survey of family practices in Virginia by Marsland et al. (6) ranked purulent otitis media 11 th. accounting for $1.7 \%$ of encounters. This ranking was the same as that found at site $E_{11}$ in 1974. No breakdown by race or age was reported in the Marsland survey.

In the Health Examination Survey of 1966-1970, white more often than black children, aged 12 to 17 years, were reported to have a history of ear infection. The differences were greater for children 6 to 11 years: $28.1 \%$ of white children were reported to have a history of earaches compared to $18.4{ }^{\prime \prime}$; of blacks, and $12.6^{\prime \prime}$ of white children had a history of running ear compared to $7.5 \%$ of black children (7).

National incidence rates of otitis media (International Classification of Diseases, adapted for use in the United States codes 381 and 382), in 1973-1974 from the Health Interview Survey of the National Center for Health Statistics, when analyzed by race showed rates for all ages were $4.1 \%$ for whites and $2.5 \%$ for blacks. The rate for white children under 17 years $(11.0 \%$ ) was twice that of black children under 17 years $(5.4 \%)(14)$. Although the differences were not as great as those found in our survey. they were substantial. and indicate that blacks were less likely to report otitis media. Racial differences in encounter rates for otitis media probably cannot be supported by differences in rates of upper respiratory infection which is often a precursor of otitis media. Health Examination Survey data indicate that black children 6 to 11 years have higher rates of colds and somewhat lower rates of sore throats and coughs than white children (7).

\section{DISCUSSION}

Differences in encounter rates for otitis media among the HMO centers related to factors other than the organization of care which was the same at all three of the sites. The differences in diagnostic style between the sites may have accounted for some, but not all, of the differences

Purulent, also known as acute or acute suppurative. otitis media is an infection of the middle ear usually caused by bacteria. Serous (secretory) otitis media is an inflammation of the middle ear believed to be caused by an allergic reaction. Purulent is distinguished from serous otitis media by the presence of purulent fluid in the middle ear. In serous otitis media cultures are usually negative for pathogenic bacteria and viruses. Chronic is usually considered to be unresolved recurrent acute otitis media. Symptoms of acute otitis media are variable, and include rhinitis, cough. hearing loss, earache, and fever. The clinical diagnosis rests upon the abnormal appearance of the tympanic membrane which is usually red, but may be grey or yellow, is usually bulging, and has impaired mobility. The clinical diagnosis of serous otitis media derives from evaluating the degree of tympanic membrane mobility in association with signs suggesting effusion, such as air bubbles, retraction, fluid level, opacity, and a white or yellow color 
(for a discussion of treatment and clinical findings distinguishing types of otitis media. see Refs. 1. 9. and 10).

In this study, at no time were there rigid diagnostic criteria or supplemental tests such as impedance audiometry, tympanocentesis, or middle ear fluid culture. Diagnoses of purulent or serous otitis media relied on clinicians' impressions of signs and symptoms and routine otoscopy. In addition to different propensities to diagnose otitis media given similar signs and symptoms, there may have been differences in the interpretation of physical tinding. particularly in regard to the color or the light reflex of the tympanic membrane. Differences in encounter rates for otitis media between the sites were greater to the extent that physicians who most readily diagnose otitis media based on signs and symptoms were also the ones who interpreted physical signs of inflammation most liberally.

Differences in encounter rates might also relate to economic class. Site $C$ is the middle site in terms of both economic class and encounter rates for otitis media. It seems unlikely that patients enrolled at site $E_{11}$ were more likely to use sources of care outside of the HMO than those at site $E_{1}$ or $C$ in view of the economic incentives associated with prepaid care.

National physician visit rates for blacks tend to be lower than for whites in all income groups (8). In an earlier HMO study, prenatal visits were begun earlier by whites than nonwhites although all care was prepaid (11). Thus, differences in use of physician services which exceed the incidence or prevalence of disease differences between blacks and whites may be due to differences in predilections to perceive. experience. acknowledge. or seek care for similar problems.

The National center for Health Statistics data suggest that racial differences associated with otitis media rates may be real. Moreover. a current prospective study of 2500 children is finding that Hispanic children have more episodes of acute otitis media than white children. and both groups have more than black children (5). Kessner et al. (4) too found rates of ear pathology almost twice as high in white as black children. Differences in encounter rates may thus be due to real differences in disease occurrence between blacks and whites, differences in the perception of ear infection. and differences in the tendency to visit a physician for ear problems. Interestingly. Shurin et al. (12) have also shown that when it has been assured that there are no differences in diagnosing otitis media and there are no apparent differences in severity or treatment. otitis media is more likely to persist in white than black children.

If blacks were suffering from untreated ear infections. one would expect them to have suffered hearing loss relative to whites. Kessner et al. (4) found the opposite to be true, as did a national survey. In the National Health Examination Survey of 19661970. eardrum abnormalities were found significantly more often among white $\left(11.9^{\prime} \%\right)$ than among black $\left(6.5^{\prime} ;\right)$ children 12 to 17 years (3). However, trouble hearing was reported more often by black than white youths.
Finding large and unexpected differences in encounter rates as those reported here raises interrelated questions: (1) What is the real difference in the rates of otitis media between blacks and whites? (2) How much variation in the encounter rates for this common disorder, which leads to prescribing expensive and potentially hazardous drugs. is due to variations in physician diagnosis? (3) What can be done to rationalize diagnostic styles? (4) Did the patients at site $E_{1}$ receive too much care, or did the patients at site $E_{I 1}$ receive too little care? (5) To what extent can providing equity in the organization of care bring about equity in terms of providing services reflecting the need for care?

\section{RIFIRINA ES ANI) NOTIS}

1. Bluestune. ( : D.. and Shurin. P. A.: Middle ear disease in children. Pediatr. (lin. N. Am.. 2l: $379(1974)$.

2. Bush. P. J., Rathin. D) L... and Spector. K. K.: Evaluation of a drug therapy protocol in an HMO. Med. ('are. $l^{-}: 566(1979)$.

3. Hearing Sensitivity and Related Medical Findings Among Youth 1217 Years. United States Vital and Health Statistics Series 11. No. 154. National (enter of Health Statistics. United States Department of Health. Education, and Welfare Publication (HRA) 76-16.36. Nowember. 1975

4. Kessner. I). M. Snow. I). ('.. and Singer. J.: Assessment of Medical (are for Children. Washington. DC: Institute of Medicine. National Academy of science. 1974.

5. Klein. J. O.: (personal communication).

6. Marsland. I). W. Wored. M.. and Mayos. F.. (ontent of tamily practice. J. Family Practice. i: 37 (1976).

7. National (enter for Health Statistes 1 xamination and Health History Findings Among (hildren and Youths, 617 vears United States. United States Department of Health. Education. and Welfare Public Health Service. Publication (HRA) 74-1611. Series II. No. 124. November 1473.

$x$. National center for Health Statistics. Physician Visits Volume and Interval Since last Visit. United States. 1971. Inited States Department of Health. Fducation. and Weltare Public Health Service No. (HRA) 75-1524. Series 10. No. 97. March. 1475

9. Olsen. A. L... Klein. S. W.. (harney. L. ct al: Prevention and therapy of serous otitis media by oral decongestant: a double-hlind study in pediatric practice. Pediatrice. 01: 674 (1978)

11. Rowe. D). S: Acute suppurative otutis media. Pediatrics. 56: 285 (1975)

11. Shapiro, S.. Weiner. L., and Densen, P. M.: ('omparison of Prematurily and perimatal mortality in a general population and in the population of a prepaid group practice. Am. J. Public llealth. 48: 170) (1958).

12. Shurin. P. A.. Pelton. S. I.. Donner. A.. and Klein. J. O.: Persistence of middleear effusion after acute otitis media in children. N. E.ngl. J. Med.. 3(k): 1121 (1979).

13. An episode of otisis media $u$ as detined as all encounters separated by al least 30 days.

14. Figures for blacks do not meet National (enter for Health Statistics standards for statistical reliability: therefore, these data are not published.

15. The authors thank Wiliam J. Rudrigues. M.D. . Ph.I). for his help with the yuestionnaire design and taan (harner. M.I).. for his helptul comments on in earlier drati.

16. Reyuests for reprints should he addressed to: Patricia J. Bush. Ph. D.. Assistant Professor. Department of Community and Family Medicine. (ieorgetown University School of Medicine. Washington. D)( 2(kn)7 (USA)

17. This research was supported hy NIH (iramt R 21-HSO)172202

18. Received for publication January 16, 1979.

19. Accepted for publication January $x$. 1980). 